\title{
MTM- and SIW-Inspired Bowtie Antenna Loaded with AMC for 5G mm-Wave Applications
}

\author{
Ayman A. Althuwayb iD \\ Electrical Engineering Department, Jouf University, Sakaka, Aljouf 72388, Saudi Arabia \\ Correspondence should be addressed to Ayman A. Althuwayb; aaalthuwayb@ju.edu.sa
}

Received 13 November 2020; Revised 17 December 2020; Accepted 5 January 2021; Published 30 January 2021

Academic Editor: Luciano Mescia

Copyright (C) 2021 Ayman A. Althuwayb. This is an open access article distributed under the Creative Commons Attribution License, which permits unrestricted use, distribution, and reproduction in any medium, provided the original work is properly cited.

\begin{abstract}
This paper investigates a feasible configuration of slotted bowtie antenna based on MTM and SIW properties for 5G millimeterwave applications. To realize the proposed slotted bowtie antenna in a compact dimension with high performances, the MTM and SIW concepts are implemented by applying the trapezoidal slots on the top surface of the antenna and metallic via holes through the substrate layer connecting the top surface to the ground plane. The antenna has been fed with a simple microstip-line which is connected to a waveguide-port. It is shown that the slotted bowtie antenna with a small dimension of $30 \times 16 \times 0.8 \mathrm{~mm}^{3}$ operates over a measured wideband of $32-34.6 \mathrm{GHz}$ with the fractional bandwidth, average gain, and radiation efficiency of $7.8 \%, 3.2 \mathrm{dBi}$, and $50 \%$, respectively. To improve the antenna's performance, the artificial magnetic conductor (AMC) properties have been employed on the ground plane by loading vertical and linear slots with various lengths. The AMC slots are aligned under the trapezoidal slots on the top surface to transfer the maximum electromagnetic signals to them for optimum radiation. The proposed method enlarges the antenna's effective aperture area, keeping constant its physical dimensions. The proposed AMCloaded antenna covers wider frequency range of $30-37 \mathrm{GHz}$ in measurement, which corresponds to $21 \%$ fractional bandwidth. The average experimental gain and radiation efficiency have been increased to $5.5 \mathrm{dBi}$ and $66.5 \%$, respectively, which illustrate the effectiveness of the proposed AMC-loaded antenna. The results confirm that the proposed slotted bowtie antenna with advantages of compact dimension, wide bandwidth, high gain and efficiency, low profile, being cost-effective, simple design, and easy fabrication process, which makes it applicable for mass production, can be a good candidate for $5 \mathrm{G}$ millimeter-wave applications.
\end{abstract}

\section{Introduction}

The demand of high data rate wireless communication has become essential, and several approaches have been presented [1-3]. Broadband antenna methods have been paid the most attention for numerous benefits, such as immunity to multipath cancellation, low interference to legacy systems, increased communication operational security, and higher data rates [4-7]. The broadband antenna methods play a significant role in the short-range wireless communication systems. One of the difficulties to realize the broadband antennas is how to model them in a compact area with required electrical specifications in the desirable band [8-10]. Additionally, planar broadband antennas have gained attention for practical applications and mass productions because of their simple layout and easier-to- integrate as well as good impedance characteristics [11-13]. To implement a planar broadband antenna, the configuration of the antenna's patch, its ground plane, and feeding mechanism are important [14]. Various techniques such as the truncated slot on the surface of the antennas or their ground plane have been demonstrated to expand their frequency band [15-17]. Several planar broadband antennas introduced in [18-20] possess small dimension. However, they suffer from low gain and radiation efficiency, which are other very important properties in antenna systems that should be considered.

Composite right/left-handed transmission lines known as metamaterial transmission lines have been studied in recent years for many applications in microwave, millimeter-wave, terahertz, and optical domains [21-23]. These kinds of transmission lines are very helpful to design 
antenna systems in a compact area with wide bandwidth and high radiation properties [24, 25]. Applications of metamaterial transmission lines in the design of antenna structures have been demonstrated in [26]. These transmission lines have implemented by four left-handed (LH) and right-handed $(\mathrm{RH})$ parameters. It is worth to mention that, the LH components are manually realizable and controllable; however, the $\mathrm{RH}$ parameters are parasitic elements which are unwantedly available in the structure. These parameters are known as series $\mathrm{LH}$ capacitor $\left(C_{L}\right)$, shunt LH inductor $\left(L_{L}\right)$, shunt $\mathrm{RH}$ capacitance $\left(C_{R}\right)$, and series $\mathrm{RH}$ inductance $\left(L_{R}\right)$ [27-29].

Besides the metamaterial technique, the substrate integrated waveguide (SIW) and artificial magnetic conductor (AMC) approaches are other interesting ideas to suppress the substrate losses and surface waves to design antenna systems with favorable properties in a simple design structure [30-37].

The frequency bandwidth from $3 \mathrm{GHz}$ to $300 \mathrm{GHz}$ known as centimeter-wave (cm-wave) and millimeter-wave (mm-wave) spectra have attracted by fifth generation (5G) technology because it can provide the data rate up to numerous gigabit-per-second (Gbps). Other reason to select this area is that the lower band's availability has formerly been utilized by many wireless systems, while most of the spectrum's upper portion is not used and can be accessible for $5 \mathrm{G}$ technology $[38,39]$.

Therefore, in this paper, three effective approaches inspired MTM, SIW, and AMC have been presented and developed to design a slotted bowtie antenna with compact dimension, wide bandwidth, and high radiation properties for application to $5 \mathrm{G}$ wireless communication systems over millimeter-wave band. First, the metamaterial approach and substrate integrated waveguide techniques are applied to the structure of a bowtie antenna to increase its effective aperture area by employing the trapezoidal slots and metallic via holes through the substrate layer connecting the top surface to the ground plane, which diminish the surface waves and substrate losses. After applying the proposed methods, it is shown that the proposed antenna can cover a wide frequency band with high radiation properties. To achieve more improvements on the antenna's performance parameters, the AMC technique has been applied to antenna's ground plane by etching the linear and vertical slots with various lengths exactly aligned underneath the trapezoidal slots to coupling maximum electromagnetic energy to the top surface to have optimum radiation. The achievements in this paper confirm the effectiveness of the proposed approaches. In the next step, the antenna's design process is discussed in detail.

\section{Slotted Bowtie Antenna Design Procedure}

Figure 1 displays the proposed bowtie antenna which has tooled, designed and fabricated on the Rogers RT5880 substrate with thickness of $0.8 \mathrm{~mm}$, dielectric constant of $\varepsilon_{r}=2.2$, and $\tan \delta=0.0009$. It is clear from Figure 1 that to realize the slotted bowtie configuration, the trapezoidal slots have been etched on the top surface of the antenna to enlarge the antenna's effective aperture area without expanding its physical dimensions. These two trapezoidal slots have been implemented in a series configuration using the properties of metamaterial transmission lines [29]. In other words, these two trapezoidal slots in a series configuration play the role of the series LH capacitances $\left(C_{L}\right)$. Besides $C_{L}$ implemented by the trapezoidal slots, another main component of the metamaterial structures is shunt LH inductance $\left(L_{L}\right)$ which is implemented here via employing the substrate integrated waveguide (SIW) concept by realizing the metallic via holes at the edge of the structure. These metallic via holes have been modeled across the Rogers RT5880 dielectric substrate layer, and they connect the top surface of the antenna to its ground plane located on the back side. The main advantage of the SIW approach is that it reduces the substrate losses and suppresses the surface waves without increasing the physical dimensions, which has led to improve the antenna's performances such as impedance matching, impedance bandwidth, and radiation specifications.

It is worth to comment that the $\mathrm{RH}$ parasitic effects such as shunt $\mathrm{RH}$ capacitances $\left(C_{R}\right)$ and series $\mathrm{RH}$ inductances $\left(L_{R}\right)$ are usually unavoidable and excised in the structure. They have been established with existence of the gap distance between the top surface and the ground plane and flowing currents on the surface, respectively [40]. Hence, the purely LH materials are not available in the nature. Therefore, the proposed metamaterial-inspired bowtie antenna is named as composite right/left-handed- (CRLH-) based bowtie antenna structure. The equivalent circuit model of the proposed metamaterial-based bowtie antenna is shown in Figure 1 to better understand its LC behavior.

The simulated layouts and fabricated prototypes of the proposed slotted bowtie antenna-inspired MTM and SIW concepts with dimensions of $30 \times 16 \times 0.8 \mathrm{~mm}^{3}$ are exhibited in Figure 1. Figure 2 shows that the proposed antenna operates over a wide frequency range between $32 \mathrm{GHz}$ and $34.6 \mathrm{GHz}$, which corresponds to $9 \%$ fractional bandwidth. Besides the frequency bandwidth, it is exhibited in Figure 3 that the minimum, maximum, and average amounts of the radiation gain and efficiency of the proposed slotted bowtie antenna are $3 \mathrm{dBi}, 3.5 \mathrm{dBi}$, and $3.2 \mathrm{dBi}$ and $48 \%, 53 \%$, and $50 \%$, respectively. The proposed antenna has excited by a simple microstrip-line which is connected to a waveguide port. The proposed antenna was designed by 3D full-wave electromagnetic CST Microwave Studio.

To improve the antenna's performance, a simple and feasible approach based on the artificial magnetic conductor (AMC) concept $[28,33]$ has been applied to the antenna's ground plane by realizing the vertical and linear slots in various lengths, as shown in Figures $1(\mathrm{c})$ and $1(\mathrm{~g})$. Besides the trapezoidal slots implemented on the top side, the AMC slots play the role of the series $\mathrm{LH}$ capacitance $\left(C_{L}\right)$ as complementary parameter to improve the left-handed (LH) properties of the antenna structure (see Figure $1(\mathrm{~h})$ ). It is obvious that by loading the AMC slots, the antenna's physical size is not changed; however, its effective aperture area is expanded, which improves the performance 


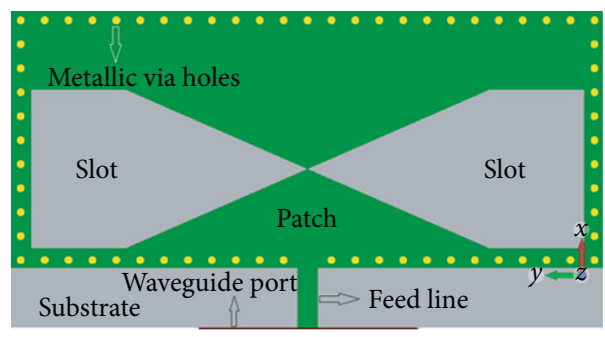

(a)

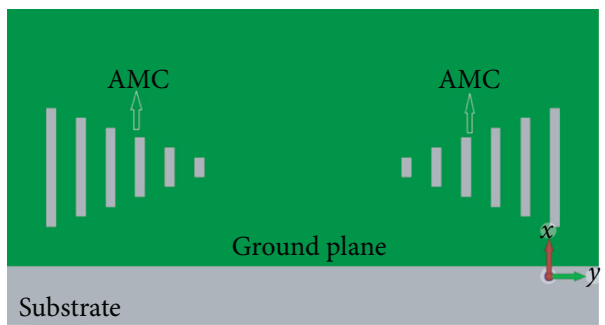

(c)

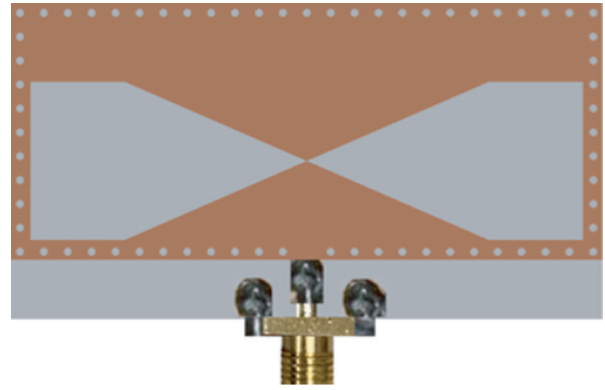

(e)

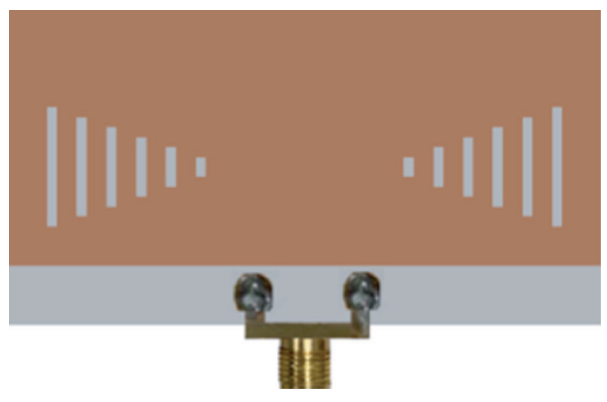

(g)

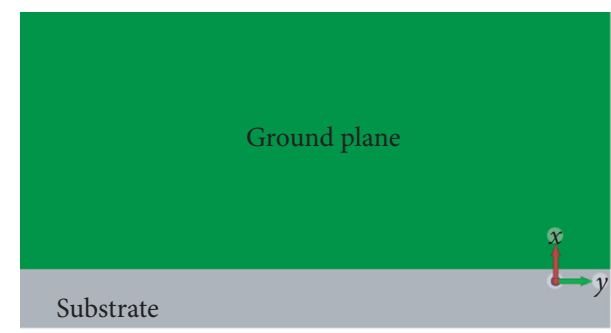

(b)

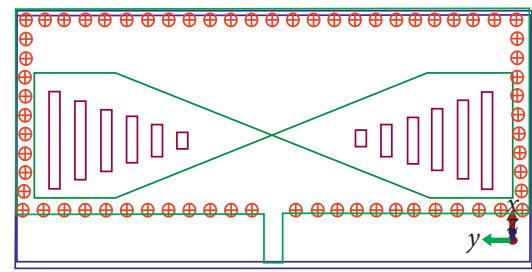

(d)

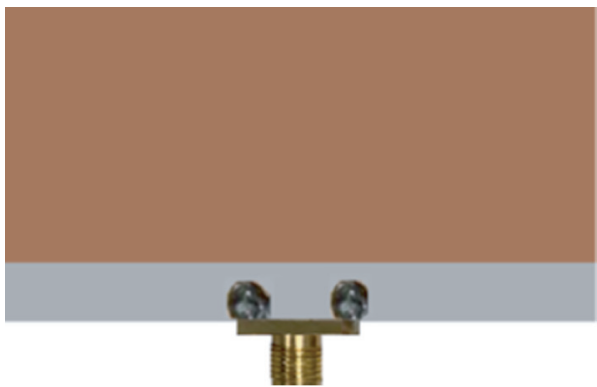

(f)

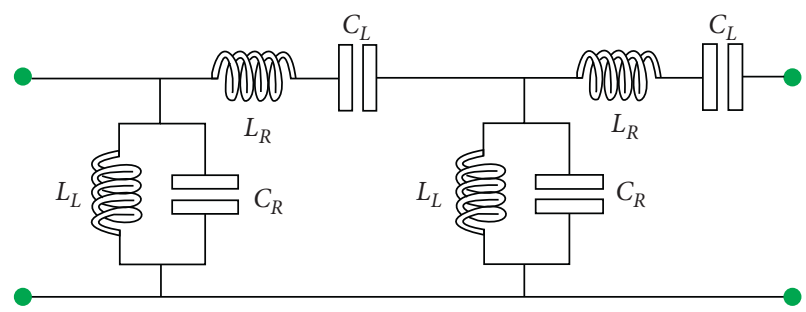

$C_{L}$ : trapezoidal slots and AMC load $L_{L}$ : via holes

(h)

FIGURE 1: Configuration of the proposed bowtie antenna structure: (a) top view of the simulated layout based on the metamaterial properties and SIW concepts; (b) ground plane without AMC; (c) ground plane loaded with AMC; (d) schematic view to show all structural components designed on the top and bottom sides; (e) fabricated prototype (top view); (f) fabricated prototype without AMC load (back view); (g) fabricated prototype loaded with AMC (back view); (h) equivalent circuit model.

parameters such as bandwidth and radiation properties, which are shown in next steps. Furthermore, to transfer maximum electromagnetic energy from the bottom side to the top side, these AMC slots have been aligned exactly underneath the trapezoidal slots to accomplish the optimum radiation. In the following parts, the effectiveness of the AMC load is exhibited and elaborated in detail. The structural parameters of the antenna were optimized by the 3D full-wave electromagnetic CST Microwave Studio and are listed in Table 1.
The simulated and measured reflection coefficient responses $\left(S_{11}<-10 \mathrm{~dB}\right)$ for both metamaterial- and SIWbased bowtie antennas without and with AMC load are plotted in Figure 2. It is obvious that the simulated and measured results for both antennas have good agreement with each other. This figure depicts that after loading the AMC slots to the ground plane, the antenna's bandwidth is expanded by $4.4 \mathrm{GHz}$, and so the AMC-loaded antenna supports the broader frequency band between $30 \mathrm{GHz}$ and $37 \mathrm{GHz}$ across the millimeter-wave area. Consequently, the 


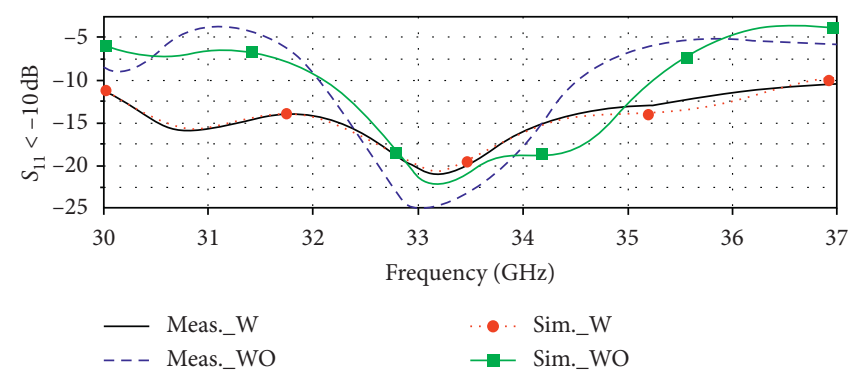

FIGURE 2: Simulated and measured reflection coefficient responses $\left(S_{11}<-10 \mathrm{~dB}\right)$ before (WO) and after (W) applying artificial magnetic conductor (AMC) load.

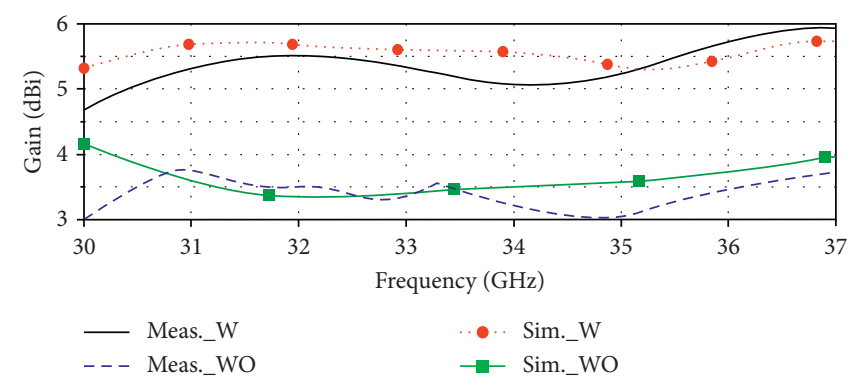

FIGURE 3: Simulated and measured radiation gain curves over the frequency band for both bowtie antennas without (WO) and with (W) AMC load.

TABle 1: Bowtie antenna's structural parameters.

\begin{tabular}{lc}
\hline Bowtie antenna dimensions & $30 \times 16 \times 0.8 \mathrm{~mm}^{3}$ \\
\hline Ground plane dimensions & $30 \times 13 \times 0.8 \mathrm{~mm}^{3}$ \\
Length of the trapezius slots & $14 \mathrm{~mm}$ \\
Maximum width of the trapezius slots & $0 \mathrm{~mm}<L \leq 8 \mathrm{~mm}$ \\
Radius of via holes & $0.2 \mathrm{~mm}$ \\
Distance between via holes & $0.4 \mathrm{~mm}$ \\
Length of AMC slots & $1 \mathrm{~mm} \leq L \leq 6 \mathrm{~mm}$ \\
Width of AMC slots & $0.5 \mathrm{~mm}$ \\
Distance between AMC slots & $1 \mathrm{~mm}$ \\
Length of feed line & $3 \mathrm{~mm}$ \\
Width of feed line & $1 \mathrm{~mm}$ \\
Substrate height & $0.8 \mathrm{~mm}$ \\
\hline
\end{tabular}

fractional bandwidth is enhanced to $21 \%$ which illustrates $13.2 \%$ improvement compared to the antenna without AMC load. Besides the frequency band, it is clear from Figure 2 that the antenna's bandwidth after AMC loading is more stable in comparison with the case without AMC load. To better understand the effectiveness of the AMC load on the antenna's bandwidth, the measured S-parameter results are summarized in Table 2.

Besides the frequency bandwidth, the radiation characteristics such as gain and efficiency are other main performance parameters that should be considered to design antenna systems. The simulated and measured radiation gain curves for both metamaterial- and SIW-inspired bowtie antennas before and after applying the AMC load are plotted in Figure 3. It is shown that the minimum, maximum, and average amounts of the radiation gain for the antenna loaded with $\mathrm{AMC}$ are $4.7 \mathrm{dBi}$,
$6.0 \mathrm{dBi}$, and $5.5 \mathrm{dBi}$, respectively, which show $1.7 \mathrm{dBi}(57 \%), 2.5$ $\mathrm{dBi}(71.5 \%)$, and $2.3 \mathrm{dBi}(72 \%)$ improvements in comparison with the bowtie antenna without AMC load. From Figure 3, it is clear that the simulated and measured results for both antennas have good coherence with each other. Additionally, after applying the AMC load, the gain curves are more stable than the case without AMC load. The radiation gain performances for both bowtie antennas without and with AMC load are summarized in Table 3.

The simulated and measured radiation efficiency curves for both bowtie antennas without and with AMC load are exhibited in Figure 4. Obviously, the simulated results have good agreement with the measured ones. It is shown that a significant improvement has been achieved after applying the AMC load across the operating bandwidth. The minimum, maximum, and average values of the radiation efficiency for the bowtie antenna loaded with AMC are 64\%, $68 \%$, and $66.5 \%$, respectively, which explain the improvements of $16 \%, 15 \%$, and $16.5 \%$ compared to the antenna without AMC load. To have a better understanding of the effectiveness of the AMC load on the radiation efficiency, Table 4 summarizes the radiation efficiency values before and after applying AMC load.

The results presented in Figures 2-4 and Tables 2-4 confirm the effectiveness of the AMC load on the antenna's performance parameters. These achievements show that the proposed slotted bowtie antenna realized based on the metamaterial, SIW, and artificial magnetic conductor (AMC) properties with the advantages of compact dimensions, wide bandwidth, high radiation gain and efficiency, low profile, being cost-effective, simple design, and easy fabrication process 
TABLE 2: Effectiveness of AMC load on the antenna's reflection coefficient responses.

\begin{tabular}{lccc}
\hline Measured frequency bandwidth & $S_{11}<-10 \mathrm{~dB}$ & Frequency band length $(\mathrm{GHz})$ & Fractional bandwidth $(\%)$ \\
\hline Antenna without AMC & $32-34.6 \mathrm{GHz}$ & 2.6 & 7.8 \\
Antenna loaded with AMC & $30-37 \mathrm{GHz}$ & 7 & 21 \\
Improvement after AMC load & & $4.4 \mathrm{GHz}$ & 13.2 \\
\hline
\end{tabular}

TABLE 3: Effectiveness of the AMC load on the radiation gain.

\begin{tabular}{lccc}
\hline Measured radiation gain & Minimum & Maximum & Average \\
\hline Antenna without AMC & $3 \mathrm{dBi}$ & $3.5 \mathrm{dBi}$ & $3.2 \mathrm{dBi}$ \\
Antenna loaded with AMC & $4.7 \mathrm{dBi}$ & $6.0 \mathrm{dBi}$ & $5.5 \mathrm{dBi}$ \\
Improvement after AMC load & $1.7 \mathrm{dBi} \cong 57 \%$ & $2.5 \mathrm{dBi} \cong 71.5 \%$ & $2.3 \mathrm{dBi} \cong 72 \%$ \\
\hline
\end{tabular}

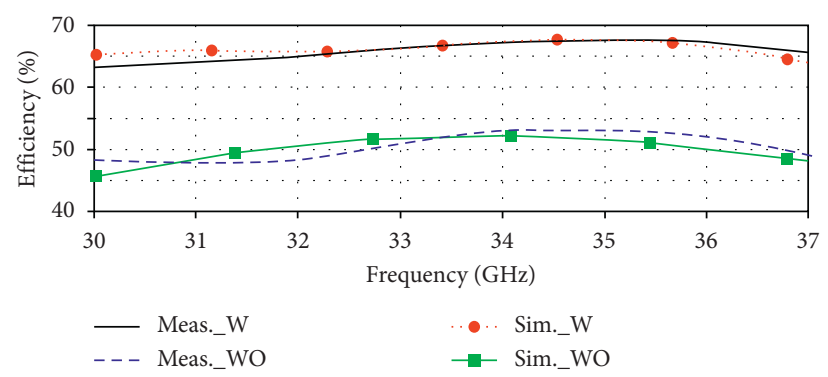

FIGURE 4: Simulated and measured radiation efficiency curves over the bandwidth for both bowtie antennas without (WO) and with (W) AMC load.

TABLE 4: Effectiveness of the AMC load on the radiation efficiency.

\begin{tabular}{lccc}
\hline Measured radiation efficiency & Minimum (\%) & Maximum (\%) & Average (\%) \\
\hline Antenna without AMC & 48 & 53 & 50 \\
Antenna loaded with AMC & 64 & 68 & 66.5 \\
Improvement after AMC load & 16 & 15 & 16.5 \\
\hline
\end{tabular}

can be used for mass production in practice, and looking at its operating frequency band and radiation properties, it is a proper candidate for applications to $5 \mathrm{G}$ wireless communication systems over millimeter-wave band.

\section{Conclusion}

Feasibility of three simple but effective approaches inspired by metamaterial (MTM), substrate integrated waveguide (SIW), artificial magnetic conductor (AMC) contents has been studied and investigated in this research work to design an innovative configuration of a slotted bowtie antenna operating from $30 \mathrm{GHz}$ to $37 \mathrm{GHz}$ that is applicable to lower band of $5 \mathrm{G}$ millimeter-wave area. To create a bowtie layout, two trapeziusshaped slots have been realized in a series configuration. It is shown that after applying the MTM and SIW principles, a wide frequency bandwidth along with high radiation performances has been achieved without affecting the physical dimensions. Then, to achieve broader bandwidth with higher radiation specifications, the AMC technique has been applied to the ground plane, keeping constant the physical size. To have optimum radiation, the AMC slots have been exactly alighted underneath two trapezoidal slots. The results show that after applying AMC load, the antenna's measured bandwidth, fractional bandwidth, gain, and radiation efficiency have improved by $4.4 \mathrm{GHz}, 13.2 \%, 2.3 \mathrm{dBi}$, and $16.5 \%$, respectively, in a compact footprint area of $30 \times 16 \times 0.8 \mathrm{~mm}^{3}$, which shows the effectiveness of the proposed approaches. Therefore, the proposed slotted bowtie antenna can be used for applications to $5 \mathrm{G}$ wireless communication systems in millimeter-wave region.

\section{Data Availability}

The data used to support the findings of this study are included within the article.

\section{Conflicts of Interest}

The author declares that there are no conflicts of interest.

\section{References}

[1] L. Rakotondrainibe, Y. Kokar, G. Zaharia, and G. El Zein, "Millimeter-wave system for high data rate indoor communications," in Proceedings of the 2009 International Symposium on Signals, Circuits and Systems, pp. 1-4, Iasi, Romania, July 2009. 
[2] M. Alibakhshikenari, B. S. Virdee, C. H. See, and P. Shukla, "Study on improvement of the performance parameters of a novel $0.41-0.47 \mathrm{THz}$ on-chip antenna based on metasurface concept realized on $50 \mu \mathrm{m}$ GaAs-layer," Scientific Reports, vol. 10, Article ID 11034, 2020.

[3] M. Alibakhshikenari, B. S. Virdee, C. H. See et al., "High-gain metasurface in polyimide on-chip antenna based on CRLHTL for sub terahertz integrated circuits," Scientific Reports, vol. 10, p. 4298, 2020.

[4] M. Alibakhshikenari, B. S. Virdee, P. Chukla et al., "Metamaterial-inspired antenna array for application in microwave breast imaging systems for tumor detection," IEEE Access, vol. 8, pp. 174667-174678, 2020.

[5] M. Alibakhshikenari, B. S. Virdee, M. Khalily et al., "Highgain on-chip antenna design on silicon layer with aperture excitation for terahertz applications," IEEE Antennas and Wireless Propagation Letters, vol. 19, no. 9, pp. 1576-1580, 2020.

[6] R. J. Fontana, "Recent system applications of short-pulse ultra-wideband (UWB) technology," IEEE Transactions on Microwave Theory and Techniques, vol. 52, no. 9, pp. 20872104, 2004.

[7] L. Li, Z.-L. Zhou, and J.-S. Hong, "Design and analysis of a novel compact wideband Antenna with two excited modes," International Journal of Antennas and Propagation, vol. 2012, Article ID 351038, 5 pages, 2012.

[8] M. Alibakhshikenari, E. Limiti, M. Naser-Moghadasi, B. S. Virdee, and R. A. Sadeghzadeh, "A new wideband planar antenna with band-notch functionality at GPS, bluetooth and WiFi bands for integration in portable wireless systems," AEUE Elsevier- International Journal of Electronics and Communications, vol. 72, pp. 79-85, 2017.

[9] T. Saeidi, I. Ismail, W. P. Wen, A. R. H. Alhawari, A. Mohammadi, and M. Ahmad, "Ultra-wideband Antennas for wireless communication applications," International Journal of Antennas and Propagation, vol. 2019, Article ID 7918765, 25 pages, 2019.

[10] C. A. Balanis, Antenna Theory: "Analysis and Design", John Wiley, Hoboken, NJ, USA, 3rd edition, 2005.

[11] A. Azari, "A new super wideband fractal microstrip antenna," IEEE Transactions on Antennas and Propagation, vol. 59, no. 5, pp. 1724-1727, 2011.

[12] S. Dey and N. C. Karmakar, "Design of novel super wide band antenna close to the fundamental dimension limit theory," Scientific Reports, vol. 10, no. 1, p. 16306, 2020.

[13] T. Okan, "A compact octagonal-ring monopole antenna for super wideband applications," Microwave and Optical Technology Letters, vol. 62, no. 3, pp. 1237-1244, 2019.

[14] S. Kumar, G. H. Lee, D. H. Kim, N. S. Haunan, H. C. Choi, and K. W. Kim, "Compact planar super-wideband monopole antenna with four notched bands," Electronics, vol. 9, no. 8, p. $1204,2020$.

[15] C. Á. Figueroa-Torres, J. L. Medina-Monroy, H. LobatoMorales, R. A. Chávez-Pérez, and A. Calvillo-Téllez, "A novel fractal antenna based on the Sierpinski structure for super wide-band applications," Microwave and Optical Technology Letters, vol. 59, no. 5, pp. 1148-1153, 2017.

[16] S. Singhal and A. K. Singh, "Modified star-star fractal (MSSF) super-wideband antenna," Microwave and Optical Technology Letters, vol. 59, no. 3, pp. 624-630, 2017.

[17] P. Okas, A. Sharma, G. Das, and R. K. Gangwar, "Elliptical slot loaded partially segmented circular monopole antenna for super wideband application," AEU-International Journal of Electronics and Communications, vol. 88, pp. 63-69, 2018.
[18] W. Balani, M. Sarvagya, T. Ali et al., "Design techniques of super-wideband antenna-existing and future prospective," IEEE Access, vol. 7, pp. 141241-141257, 2019.

[19] S. U. Rahman, Q. Cao, H. Ullah, and H. Khalil, "Compact design of trapezoid shape monopole antenna for SWB application," Microwave and Optical Technology Letters, vol. 61, no. 8, pp. 1931-1937, 2019.

[20] S. Singhal, "Asymmetrically fed octagonal Sierpinski bandnotched super-wideband antenna," Journal of Computational Electronics, vol. 16, no. 1, pp. 210-219, 2017.

[21] M. Mohammadi, F. H. Kashani, and J. Ghalibafan, "Backfireto-endfire scanning capability of a balanced metamaterial structure based on slotted ferrite-filled waveguide," Waves Random Complex Media, pp. 1-15, 2019.

[22] C. Caloz and T. Itoh, Electromagnetic Metamaterials: Transmission Line Theory and Microwave Applications, WileyIEEE Press, Hoboken, NJ, USA, 2005.

[23] M. Alibakhshikenari, B. S. Virdee, L. Azpilicueta et al., "A comprehensive survey of "metamaterial transmission-line based antennas: design, challenges, and applications"” IEEE Access, vol. 8, pp. 144778-144808, 2020.

[24] M. Shirkolaei and J. Ghalibafan, "Unbalanced CRLH behavior of ferrite-loaded waveguide operated below cutoff frequency," Waves in Random and Complex Media, 2020.

[25] M. Alibakhshi-Kenari, M. Naser-Moghadasi, and R. A. Sadeghzadeh, "Composite right-left-handed-based antenna with wide applications in very-high frequency-ultrahigh frequency bands for radio transceivers," IET Microwaves, Antennas \& Propagation, vol. 9, no. 15, pp. 1713-1726, 2015.

[26] K. L. Chung, "High-performance circularly polarized antenna array using metamaterial-line based feed network," IEEE Transactions on Antennas and Propagation, vol. 61, no. 12, pp. 6233-6237, 2013.

[27] M. Mohammadi, F. H. Kashani, and J. Ghalibafan, “A partially ferrite-filled rectangular waveguide with CRLH response and its application to a magnetically scannable antenna," Journal of Magnetism and Magnetic Materials, vol. 491, Article ID 165551, 2019.

[28] M. Alibakhshi-Kenari, M. Naser-Moghadasi, R. A. Sadeghzadeh et al., "Bandwidth and radiation specifications enhancement of monopole antennas loaded with split ring resonators," IET Microwaves, Antennas \& Propagation, vol. 9, no. 14, pp. 14871496, 2015.

[29] K. L. Chung, X. Yan, S. Chaimool, B. Feng, and Y. Li, "On the surface susceptibilities and effective parameters of metasurfaces composed of isolated metallic unit-cells," International Journal of RF and Microwave Computer-Aided Engineering, vol. 31, no. 2, 2020.

[30] K. Kumar, S. Dwari, and S. Priya, "Dual band dual polarized cavity backed cross slot half mode substrate integrated waveguide antenna," in Proceedings of the 2017 IEEE Applied Electromagnetics Conference (AEMC), pp. 1-2, Aurangabad, India, December 2017.

[31] M. Alibakhshikenari, B. S. Virdee, C. H. See et al., "Beamscanning leaky-wave antenna based on CRLH-metamaterial for millimeter-wave applications," IET Microwaves, Antennas \& Propagation, vol. 13, no. 8, pp. 1129-1133, 2019.

[32] R. Bayderkhani, K. Forooraghi, and B. Abbasi-Arand, "Gainenhanced SIW cavity-backed slot antenna with arbitrary levels of inclined polarization," IEEE Antennas and Wireless Propagation Letters, vol. 14, pp. 931-934, 2015.

[33] M. Alibakhshikenari, B. S. Virdee, M. Khalily et al., "New approach to suppress mutual coupling between longitudinalslotted arrays based on SIW antenna loaded with metal-fences 
working on VHF/UHF frequency-bands: study, investigation, and principle," in Proceedings of the 2018 Asia-Pacific Microwave Conference (APMC), pp. 1564-1566, Kyoto, Japan, November 2018.

[34] S. Mukherjee and A. Biswas, "Design of dual band and dualpolarised dual band SIW cavity backed bow-tie slot antennas," IET Microwaves, Antennas \& Propagation, vol. 10, no. 9, pp. 1002-1009, 2016.

[35] M. Alibakhshikenari, B. S. Virdee, C. H. See et al., "Mutualcoupling reduction in metamaterial substrate integrated waveguide slotted antenna arrays using metal fence isolators for SAR and MIMO applications," in Proceedings of the 2018 12th International Congress on Artificial Materials for Novel Wave Phenomena (Metamaterials), pp. 13-15, Espoo, Finland, August 2018.

[36] M. Mbaye, J. Hautcoeur, L. Talbi, and K. Hettak, "Bandwidth broadening of dual-slot antenna using substrate integrated waveguide (SIW)," IEEE Antennas and Wireless Propagation Letters, vol. 12, pp. 1169-1171, 2013.

[37] M. Alibakhshi-Kenari, M. Naser-Moghadasi, R. A. Sadeghzadeh, B. S. Virdee, and E. Limiti, "Periodic array of complementary artificial magnetic conductor metamaterials-based multiband Antennas for broadband wireless transceivers," IET Microwaves, Antennas \& Propagation, vol. 10, no. 15, pp. 1682-1691, 2016.

[38] B. Feng, Y. Tu, J. Chen, S. Yin, and K. L. Chung, "Dual linearly-polarized antenna array with high gain and high isolation for $5 \mathrm{G}$ millimeter-wave applications," IEEE Access, vol. 8, pp. 82471-82480, 2020.

[39] D. A. Sehrai, M. Abdullah, A. Altaf et al., "A novel high gain wideband MIMO antenna for $5 \mathrm{G}$ millimeter wave applications," Electronics, vol. 9, no. 6, p. 1031, 2020.

[40] B. Feng, J. Lai, Q. Zeng, and K. L. Chung, "A dual-wideband and high gain magneto-electric dipole antenna and its 3D MIMO system with metasurface for 5G/WiMAX/WLAN/XBand Applications," IEEE Access, vol. 6, pp. 33387-33398, 2018. 